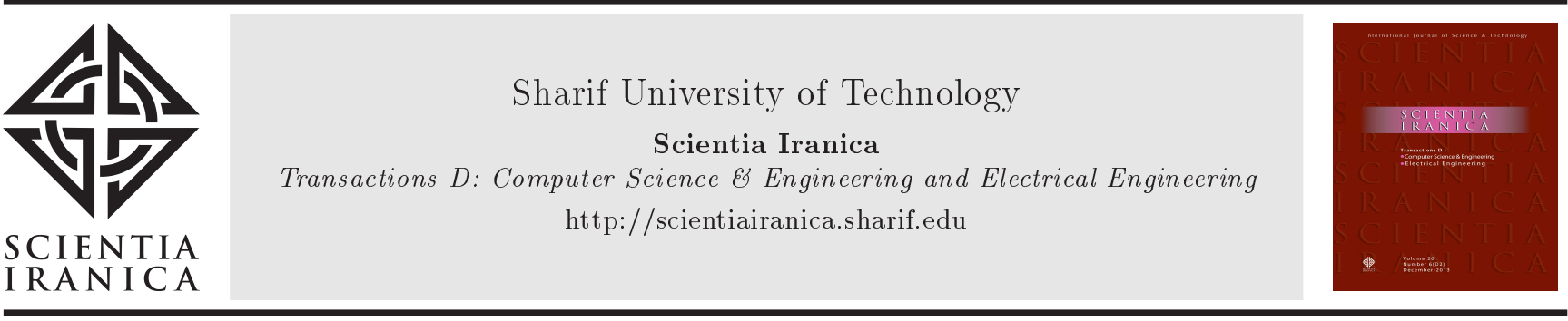

\title{
A new approximate analytical method and its convergence for nonlinear time-fractional partial differential equations
}

\author{
A. Khalouta* and A. Kadem \\ Laboratory of Fundamental and Numerical Mathematics, Department of Mathematics, Faculty of Sciences, Ferhat Abbas Sétif \\ University 1, 19000 Sétif, Algeria.
}

Received 1 February 2020; received in revised form 25 November 2020; accepted 8 February 2021

\section{KEYWORDS \\ Fractional model; Riemann-Liouville integral; \\ Caputo derivative; \\ Numerical method; \\ Approximate \\ analytical solution.}

\begin{abstract}
The main objective of this paper is to present a new approximate analytical method called Modified Generalized Taylor Fractional Series Method (MGTFSM) for solving general nonlinear time-fractional partial differential equations. The fractional derivative is considered in the Caputo sense. The convergence results of the proposed method are established here. The basic idea of the MGTFSM is to construct the solution in the form of infinite series that converges rapidly to the exact solution of the given problem. The main advantage of the proposed method, compared to current methods, is that the method solves the nonlinear problems without using linearization, discretization, perturbation, or any other restriction. The efficiency and accuracy of the MGTFSM are tested by means of different numerical examples. The results prove that the proposed method is very effective and simple for solving the nonlinear time-fractional partial differential equations problems.

(C) 2021 Sharif University of Technology. All rights reserved.
\end{abstract}

\section{Introduction}

In recent years, many mathematicians and physicists have been interested in the subject of Nonlinear Fractional Partial Differential Equations (NFPDEs) because of their many applications in various fields where they can be used for modeling a wide range of physical phenomena such as statistical mechanics, mathematical physics, theoretical neuroscience, fluid dynamics, electrochemistry, viscoelasticity, population dynamics, cancer modeling, and mathematical finance. So, the theory of NFPDEs is a powerful theory to give a solution to engineering problems (see, e.g., [1-15]).

\footnotetext{
*. Corresponding author. Tel.: +213 779447558 E-mail addresses: nadjibkh@yahoo.fr (A. Khalouta); abdelouahabk@yahoo.fr (A. Kadem)
}

Obtaining approximate analytical solutions to the NFPDEs is one of the very important subjects in mathematics, science, and technology. For this purpose, many different numerical and analytical methods have been constructed and developed, among which Modification of Adomian Decomposition Method (MADM) [16], Variational Iteration Method (VIM) [17], Homotopy Analysis Method (HAM) [18], Homotopy Perturbation Transform Method (HPTM) [19], New Iterative Method (NIM) [20], Fractional Elzaki Projected Differential Transform Method (FEPDTM) [21], Generalized Differential Transform Method (GDTM) [22], Modification of the Reduced Differential Transform Method (MRDTM) [23], Fractional Taylor Operational Matrix Method (FTOMM) [24], and Fractional Residual Power Series Method (FRPSM) [25].

The Modified Generalized Taylor Fractional Series Method (MGTFSM) was first proposed to obtain 
a numerical solution to a certain class of NFPDEs, see [26]. The MGTFSM consists of an iterative algorithm. This method is effective and easy to obtain a power series solution to linear and nonlinear fractional differential equations without resorting to linearization, perturbation, or discretization. Unlike other series methods, the MGTFSM does not match the coefficients of the similar conditions and a repeated connection is not required. The present method computes the coefficients of the power series by a bond of algebraic equations of some variables. In addition, the MGTFSM does not need any transformation during the change from low to higher order; thus, it is possible to work with the present method directly on the given example by choosing a suitable initial estimate approximation. This method has been tested to be powerful, effective, and easily capable to deal with a wide range of linear and nonlinear fractional differential equations. The main objective of this paper is to extend this method and establish its convergence to solve general nonlinear time-fractional partial differential equations with arbitrary nonlinear terms.

The rest of the paper is organized as follows. Section 2 presents some basic definitions and properties of fractional calculus. Section 3 introduces our obtained results in the form of a new theorems to solve the general nonlinear time-fractional partial differential equations by using the MGTFSM. Section 4 gives some numerical examples to exhibit the efficiency and effectiveness of the proposed method. Section 5 discusses our obtained results represented by figures and tables. Section 6 summarizes the conclusions of this work.

\section{Basic definitions of fractional calculus}

In this section, some basic definitions and properties of fractional calculus, which are used further in this paper, are introduced. For more details, see [27,28].

Definition 1. A real function $f(t), t>0$, is considered to be in the space $\mathrm{C}_{\mu}([0, \infty]), \mu \in \mathbb{R}$ if there exists a real number $p>\mu$ so that $f(t)=t^{p} h(t)$, where $h$ $\in C([0, \infty])$, and it is said to be in the space $\mathrm{C}_{\mu}^{n}$ if $f^{(n)} \in \mathrm{C}_{\mu}([0, \infty]), n \in \mathbb{N}$.

Definition 2. The Riemann-Liouville fractional integral of order $\alpha \geq 0$ of a function $f \in \mathrm{C}_{\mu}([0, \infty]), \mu \geq$ -1 , is defined as follows:

$$
I^{\alpha} f(t)=\frac{1}{\Gamma(\alpha)} \int_{0}^{t}(t-\xi)^{\alpha-1} f(\xi) d \xi, \quad \alpha>0, \quad t>0,
$$

where $\Gamma($.$) is the well-known Gamma function.$

Definition 3. The Caputo fractional derivative of order $n-1<\alpha \leq n$ of a function $f \in \mathrm{C}_{-1}^{n}([0, \infty]), n \in$
$\mathbb{N}$, is defined as

$$
D^{\alpha} f(t)=\frac{1}{\Gamma(n-\alpha)} \int_{0}^{t}(t-\xi)^{n-\alpha-1} f^{(n)}(\xi) d \xi .
$$

For this definition, we have the following properties:

$$
D^{\alpha}(C)=0,
$$

where $C$ is a constant.

$$
\begin{aligned}
& D^{\alpha} t^{\beta}= \begin{cases}\frac{\Gamma(\beta+1)}{\Gamma(\beta-\alpha+1)} t^{\beta-\alpha}, & \text { if } \beta>n-1, \\
0, & \text { if } \beta \leq n-1 .\end{cases} \\
& D^{\alpha}[\lambda f(t)+\nu g(t)]=\lambda D^{\alpha}[f(t)]+\nu D^{\alpha}[g(t)], \\
& (\lambda, \nu) \in \mathbb{R}^{2}, \\
& D^{\alpha}[f(t) g(t)]=g(t) D^{\alpha}[f(t)]+f(t) D^{\alpha}[g(t)] .
\end{aligned}
$$

Definition 4. The Caputo time-fractional derivative of order $n-1<\alpha \leq n$ of a function $u \in \mathrm{C}_{-1}^{n}(\mathbb{R} \times$ $[0, \infty]), n \in \mathbb{N}$, is defined as:

$$
D_{t}^{\alpha} u(x, t)=\frac{1}{\Gamma(n-\alpha)} \int_{0}^{t}(t-\xi)^{n-\alpha-1} \frac{\partial^{n} u(x, \xi)}{\partial \xi^{n}} d \xi .
$$

Definition 5. The Mittag-Leffler function is defined as follows:

$$
E_{\alpha}(z)=\sum_{n=0}^{\infty} \frac{z^{n}}{\Gamma(n \alpha+1)}, \quad \alpha>0, \quad z \in \mathbb{C} .
$$

For $\alpha=1, E_{\alpha}(z)$ reduces to $e^{z}$.

\section{Analytical study of MFTSM}

Theorem 1. Consider a general nonlinear timefractional partial differential equation:

$$
\begin{gathered}
D_{t}^{\alpha} u(x, t)=\mathcal{L}(u(x, t))+\mathcal{N}(u(x, t)), \\
x \in I \subset \mathbb{R}, t \geq 0,
\end{gathered}
$$

with the initial conditions:

$$
u(x, 0)=u_{0}(x),
$$

where $D_{t}^{\alpha}$ is the Caputo fractional derivative of order $\alpha, 0<\alpha \leq 1, \mathcal{L}(u(x, t))$ is a linear differential operator, and $\mathcal{N}(u(x, t))$ represents a general nonlinear differential operator. Then, by MGTFSM, the solution to Eqs. (9)-(10) is given in the form of infinite series as follows:

$$
\begin{aligned}
u(x, t) & =\sum_{j=0}^{\infty} u_{j}(x) \frac{t^{j \alpha}}{\Gamma(j \alpha+1)}, \\
x & \in I \subset \mathbb{R}, \quad 0 \leq t<R,
\end{aligned}
$$

where $u_{j}(x)$ is the coefficient of Series (11) and $R$ is the radius of convergence. 
Proof. In order to achieve our goal, we consider the following generalized nonlinear time-fractional partial differential equation (9) with the initial conditions (10). by:

We assume the solution as an infinite series given

$$
u(x, t)=\sum_{j=0}^{\infty} u_{j}(x) \frac{t^{j \alpha}}{\Gamma(j \alpha+1)} .
$$

Consequently, the approximate solution of Eqs. (9) and (10) can be written as follows:

$$
\begin{aligned}
u_{n}(x, t) & =\sum_{j=0}^{n} u_{j}(x) \frac{t^{j \alpha}}{\Gamma(j \alpha+1)} \\
& =u_{0}(x)+\sum_{j=1}^{n} u_{j}(x) \frac{t^{j \alpha}}{\Gamma(j \alpha+1)} .
\end{aligned}
$$

By applying the operator $D_{t}^{\alpha}$ to Eq. (13) and using Properties (1) and (2), the following formula is obtained:

$$
D_{t}^{\alpha} u_{n}(x, t)=\sum_{j=0}^{n-1} u_{j+1}(x) \frac{t^{j \alpha}}{\Gamma(j \alpha+1)} .
$$

We substitute Eqs. (13) and (14) into Eq. (9) and obtain the following recurrence relation:

$$
\begin{aligned}
0= & \sum_{j=0}^{n-1} u_{j+1}(x) \frac{t^{j \alpha}}{\Gamma(j \alpha+1)}-\mathcal{L}\left(\sum_{j=0}^{n} u_{j}(x) \frac{t^{j \alpha}}{\Gamma(j \alpha+1)}\right) \\
& \quad-\mathcal{N}\left(\sum_{j=0}^{n} u_{j}(x) \frac{t^{j \alpha}}{\Gamma(j \alpha+1)}\right)
\end{aligned}
$$

To find the functions $u_{n}(x), n=1,2,3, .$. we follow the same methodology to obtain the coefficients of the Taylor series; therefore, we need to solve the following equation:

$$
D_{t}^{(n-1) \alpha}\{G(x, t, \alpha, n)\} \downarrow_{t=0}=0,
$$

where:

$$
\begin{gathered}
G(x, t, \alpha, n)=\sum_{j=0}^{n-1} u_{j+1}(x) \frac{t^{j \alpha}}{\Gamma(j \alpha+1)} \\
-\mathcal{L}\left(\sum_{j=0}^{n} u_{j}(x) \frac{t^{j \alpha}}{\Gamma(j \alpha+1)}\right) \\
-\mathcal{N}\left(\sum_{j=0}^{n} u_{j}(x) \frac{t^{j \alpha}}{\Gamma(j \alpha+1)}\right) .
\end{gathered}
$$

Now, we calculate the first terms of the sequence $\left\{u_{n}(x)\right\}_{1}^{N}$. For $n=1$, we have:

$$
\begin{aligned}
G(x, t, \alpha, 1)= & u_{1}(x)-\mathcal{L}\left(u_{0}(x)+u_{1}(x) \frac{t^{\alpha}}{\Gamma(\alpha+1)}\right) \\
& -\mathcal{N}\left(u_{0}(x)+u_{1}(x) \frac{t^{\alpha}}{\Gamma(\alpha+1)}\right) \cdot
\end{aligned}
$$

Solution to the equation $G(x, t, \alpha, 1)=0$ gives:

$$
u_{1}(x)=\mathcal{L}\left(u_{0}(x)\right)+\mathcal{N}\left(u_{0}(x)\right) .
$$

To find $u_{2}(x)$, we consider $G(x, t, \alpha, 2)$ and solve:

$$
D_{t}^{\alpha}\{G(x, t, \alpha, 2)\} \downarrow_{t=0}=0 .
$$

To find $u_{3}(x)$, we consider $G(x, t, \alpha, 3)$ and solve:

$$
D_{t}^{2 \alpha}\{G(x, t, \alpha, 3)\} \downarrow_{t=0}=0 .
$$

To find $u_{4}(x)$, we consider $G(x, t, \alpha, 4)$ and solve:

$$
D_{t}^{3 \alpha}\{G(x, t, \alpha, 4)\} \downarrow_{t=0}=0,
$$

and so on.

In general, to obtain the coefficient function $u_{k}(x)$, we solve:

$$
D_{t}^{(k-2) \alpha}\{G(x, t, \alpha, k)\} \downarrow_{t=0}=0,
$$

where:

$$
\begin{array}{r}
G(x, t, \alpha, k)=\sum_{j=0}^{k-1} u_{j+1}(x) \frac{t^{j \alpha}}{\Gamma(j \alpha+1)} \\
-\mathcal{L}\left(\sum_{j=0}^{k} u_{j}(x) \frac{t^{j \alpha}}{\Gamma(j \alpha+1)}\right) \\
-\mathcal{N}\left(\sum_{j=0}^{k} u_{j}(x) \frac{t^{j \alpha}}{\Gamma(j \alpha+1)}\right) .
\end{array}
$$

Finally, the solution of Eqs. (9) and (10) can be expressed as follows:

$$
\begin{aligned}
u(x, t) & =\lim _{n \rightarrow \infty} u_{n}(x, t)=\lim _{n \rightarrow \infty} \sum_{j=0}^{n} u_{j}(x) \frac{t^{j \alpha}}{\Gamma(j \alpha+1)} \\
& =\sum_{j=0}^{\infty} u_{j}(x) \frac{t^{j \alpha}}{\Gamma(j \alpha+1)} .
\end{aligned}
$$

The proof is complete.

Theorem 2. If there exists a constant $0<\gamma<1$ such that:

$$
\begin{aligned}
& \left\|u_{n+1}(x, t)\right\| \leq \gamma\left\|u_{n}(x, t)\right\|, \\
& n \in \mathbb{N}, \quad x \in I \subset \mathbb{R}, \quad 0 \leq t<R,
\end{aligned}
$$

then the sequence of approximate solution (11) converges to the exact solution. 
Proof. For all $x \in I \subset \mathbb{R}, 0 \leq t<R$, we have:

$$
\begin{aligned}
\| u(x, t) & -u_{n}(x, t)\|=\| \sum_{j=n+1}^{\infty} u_{j}(x, t) \| \\
\leq & \sum_{j=n+1}^{\infty}\left\|u_{j}(x, t)\right\| \leq \sum_{j=n+1}^{\infty} \gamma\left\|u_{j-1}(x, t)\right\| \\
\leq & \sum_{j=n+1}^{\infty} \gamma^{2}\left\|u_{j-2}(x, t)\right\| \leq \ldots \\
\leq & \left\|u_{0}(x)\right\| \sum_{j=n+1}^{\infty} \gamma^{j}=\frac{\gamma^{n+1}}{1-\gamma}\left\|u_{0}(x)\right\| .
\end{aligned}
$$

Since $0<\gamma<1$ and $u_{0}(x)$ is bounded, we have:

$$
\lim _{n \longrightarrow \infty}\left\|u(x, t)-u_{n}(x, t)\right\|=0 .
$$

This completes the proof. $\square$

\section{Applications of MGTFSM to nonlinear time-fractional partial differential equations}

In this section, some numerical examples are given to exhibit the efficiency and effectiveness of the MGTFSM in solving nonlinear time-fractional partial differential equations.

Example 1. We consider the cubic nonlinear timefractional Schrödinger equation [29]:

$$
i D_{t}^{\alpha} u=-u_{x x}+2|u|^{2} u
$$

with the initial condition:

$$
u(x, 0)=e^{i x},
$$

where $D_{t}^{\alpha}$ is the Caputo time-fractional derivative of order $\alpha, 0<\alpha \leq 1, u$ is a complex function of $(x, t) \in$ $\mathbb{R} \times \mathbb{R}^{+}$, and $i^{2}=-1$.

For $\alpha=1$, the exact solution of Eqs. (29) and (30) is given by:

$$
u(x, t)=e^{i(x-3 t)} .
$$

By applying the steps involved in the MGTFSM as presented in Section 3, we have the solution of Eqs. (29) and (30) in the form:

$$
u(x, t)=\sum_{i=0}^{\infty} u_{j}(x) \frac{t^{j \alpha}}{\Gamma(j \alpha+1)},
$$

and the coefficients of Series (32) are given as follows:

$$
u_{j}(x)=(-1)^{j}(3 i)^{j} e^{i x} \quad \text { for } \quad j=0,1,2,3, \ldots
$$

Therefore, the solution of Eqs. (29) and (30) can be expressed as follows:

$$
\begin{aligned}
& u(x, t)=e^{i x} \\
& \left(1-\frac{\left(3 i t^{\alpha}\right)}{\Gamma(\alpha+1)}+\frac{\left(3 i t^{\alpha}\right)^{2}}{\Gamma(2 \alpha+1)}-\frac{\left(3 i t^{\alpha}\right)^{3}}{\Gamma(3 \alpha+1)}+\cdots\right) \\
& =e^{i x} \sum_{j=0}^{\infty} \frac{(-1)^{j}\left(3 i t^{\alpha}\right)^{j}}{\Gamma(j \alpha+1)} \\
& =e^{i x} E_{\alpha}\left(-3 i t^{\alpha}\right),
\end{aligned}
$$

where $E_{\alpha}\left(-3 i t^{\alpha}\right)$ is the Mittag-Leffler function defined by Eq. (8). Taking $\alpha=1$ to Eq. (34) will bear the following result:

$$
\begin{aligned}
u(x, t) & =e^{i x}\left(1-3 i t+\frac{(3 i t)^{2}}{2 !}-\frac{(3 i t)^{3}}{3 !}+\cdots\right) \\
& =e^{i(x-3 t)},
\end{aligned}
$$

which is the exact solution to the classical Schrödinger equation.

Example 2. We consider the nonlinear time-fractional gas dynamic equation [30]:

$$
D_{t}^{\alpha} u=-u u_{x}+u(1-u),
$$

with the initial condition

$$
u(x, 0)=e^{-x},
$$

where $D_{t}^{\alpha}$ is the Caputo time-fractional derivative of order $\alpha, 0<\alpha \leq 1$, and $u$ is a function of $(x, t) \in$ $\mathbb{R} \times \mathbb{R}^{+}$.

For $\alpha=1$, the exact solution of Eqs. (36) and (37) is given by:

$$
u(x, t)=e^{t-x} .
$$

$B y$ applying the steps involved in the MGTFSM as presented in Section 3, we have the solution of Eqs. (36) and (37) in the form:

$$
u(x, t)=\sum_{j=0}^{\infty} u_{j}(x) \frac{t^{j \alpha}}{\Gamma(j \alpha+1)},
$$

and the coefficients of Series (39) are given as follows:

$$
u_{j}(x)=e^{-x} \quad \text { for } \quad j=0,1,2,3, \ldots
$$

Therefore, the solution of Eqs. (36) and (37) can be expressed as follows:

$$
\begin{aligned}
& u(x, t)=e^{-x} \\
& \left(1+\frac{t^{\alpha}}{\Gamma(\alpha+1)}+\frac{t^{2 \alpha}}{\Gamma(2 \alpha+1)}+\frac{t^{3 \alpha}}{\Gamma(3 \alpha+1)}+\cdots\right) \\
& =e^{-x} \sum_{j=0}^{\infty} \frac{t^{j \alpha}}{\Gamma(j \alpha+1)}=e^{-x} E_{\alpha}\left(t^{\alpha}\right),
\end{aligned}
$$


where $E_{\alpha}\left(t^{\alpha}\right)$ is the Mittag-Leffler function defined by Eq. (8). Taking $\alpha=1$ in Eq. (41) will give the following result:

$$
u(x, t)=\left(1+t+\frac{t^{2}}{2 !}+\frac{t^{3}}{3 !}+\cdots\right) e^{-x}=e^{t-x},
$$

which is the exact solution of classical gas dynamic equation.

Example 3. We consider the nonlinear timefractional reaction-diffusion-convection equation [31]:

$$
D_{t}^{\alpha} u=u_{x x}-u_{x}+u u_{x}-u^{2}+u,
$$

with the initial condition:

$$
u(x, 0)=e^{x},
$$

where $D_{t}^{\alpha}$ is the Caputo time-fractional derivative of order $\alpha, 0<\alpha \leq 1$ and $u$ is a function of $(x, t) \in$ $\mathbb{R} \times \mathbb{R}^{+}$.

For $\alpha=1$, the exact solution of Eqs. (43) and (44) is given by:

$$
u(x, t)=e^{x+t} .
$$

By applying the steps involved in the MGTFSM as presented in Section 3, we have the solution of Eqs. (43) and (44) in the form:

$$
u(x, t)=\sum_{j=0}^{\infty} u_{j}(x) \frac{t^{j \alpha}}{\Gamma(j \alpha+1)},
$$

and the coefficients of Series (46) are given as follows:

$$
u_{j}(x)=e^{x} \quad \text { for } \quad j=0,1,2,3, \ldots
$$

Therefore, the solution of Eqs. (43) and (44), can be expressed as follows:

$$
\begin{aligned}
& u(x, t)=e^{x} \\
& \left(1+\frac{t^{\alpha}}{\Gamma(\alpha+1)}+\frac{t^{2 \alpha}}{\Gamma(2 \alpha+1)}+\frac{t^{3 \alpha}}{\Gamma(3 \alpha+1)}+\cdots\right) \\
& =e^{x} \sum_{i=0}^{\infty} \frac{t^{j \alpha}}{\Gamma(j \alpha+1)}=e^{x} E_{\alpha}\left(t^{\alpha}\right),
\end{aligned}
$$

where $E_{\alpha}\left(t^{\alpha}\right)$ is the Mittag-Leffler function, defined by Eq. (8). Taking $\alpha=1$ in Eq. (48) will give the following result:

$$
u(x, t)=e^{x}\left(1+t+\frac{t^{2}}{2 !}+\frac{t^{3}}{3 !}+\cdots\right)=e^{x+t},
$$

which is the exact solution of classical reactiondiffusion-convection equation.

Example 4. Consider the nonlinear time-fractional Fokker-Planck equation [29]:

$$
D_{t}^{\alpha} u=\left(\frac{x}{3} u\right)_{x}-\left(\frac{4}{x} u^{2}\right)_{x}+\left(u^{2}\right)_{x x}
$$

with the initial condition:

$$
u(x, 0)=x^{2},
$$

where $D_{t}^{\alpha}$ is the Caputo time-fractional derivative of order $\alpha, 0<\alpha \leq 1$ and $u$ is a function of $(x, t) \in$ $\mathbb{R} \times \mathbb{R}^{+}$.

For $\alpha=1$, the exact solution of Eqs. (50) and (51) is given by:

$$
u(x, t)=x^{2} e^{t}
$$

By applying the steps involved in the MGTFSM as presented in Section 3, we have the solution of Eqs. (50) and (51) in the form:

$$
u(x, t)=\sum_{i=0}^{\infty} u_{j}(x) \frac{t^{j \alpha}}{\Gamma(j \alpha+1)},
$$

and the coefficients of Series (53) are given as follows:

$$
u_{j}(x)=x^{2} \quad \text { for } \quad j=0,1,2,3, \ldots
$$

Therefore, the solution of Eqs. (50) and (51) can be expressed as follows:

$$
\begin{aligned}
& u(x, t)=x^{2} \\
& \left(1+\frac{t^{\alpha}}{\Gamma(\alpha+1)}+\frac{t^{2 \alpha}}{\Gamma(2 \alpha+1)}+\frac{t^{3 \alpha}}{\Gamma(3 \alpha+1)}+\cdots\right) \\
& =x^{2} \sum_{j=0}^{\infty} \frac{t^{j \alpha}}{\Gamma(j \alpha+1)}=x^{2} E_{\alpha}\left(t^{\alpha}\right)
\end{aligned}
$$

where $E_{\alpha}\left(t^{\alpha}\right)$ is the Mittag-Leffler function, defined by Eq. (8). Taking $\alpha=1$ in Eq. (55) will give the following result:

$$
u(x, t)=x^{2}\left(1+t+\frac{t^{2}}{2 !}+\frac{t^{3}}{3 !}+\cdots\right)=x^{2} e^{t},
$$

which is the exact solution to the classical FokkerPlanck equation.

Example 5. Consider the 2-dimensional nonlinear time-fractional biological population equation [32]:

$$
D_{t}^{\alpha} u=\left(u^{2}\right)_{x x}+\left(u^{2}\right)_{y y}-u\left(1+\frac{8}{9} u\right)
$$

with the initial condition:

$$
u(x, y, 0)=e^{(x+y) / 3},
$$


where $D_{t}^{\alpha}$ is the Caputo time-fractional derivative of order $\alpha, 0<\alpha \leq 1$ and $u$ is a function of $(x, y, t) \in$ $\mathbb{R} \times \mathbb{R} \times \mathbb{R}^{+}$.

For $\alpha=1$, the exact solution of Eqs. (57) and (58) is given by:

$$
u(x, y, t)=e^{(1 / 3)(x+y)-t} .
$$

By applying the steps involved in the MGTFSM as presented in Section 3, we have the solution of Eqs. (57) and (58) in the form:

$$
u(x, y, t)=\sum_{i=0}^{\infty} u_{i}(x, y) \frac{t^{i \alpha}}{\Gamma(i \alpha+1)},
$$

and the coefficients of Series (60) are given as follows:

$$
u_{i}(x, y)=(-1)^{i} e^{(x+y) / 3} \text { for } i=0,1,2,3, \cdots
$$

Therefore, the solution of Eqs. (57) and (58) can be expressed as follows:

$$
\begin{aligned}
& u(x, y, t)=e^{(x+y) / 3} \\
& \left(1-\frac{t^{\alpha}}{\Gamma(\alpha+1)}+\frac{t^{2 \alpha}}{\Gamma(2 \alpha+1)}-\frac{t^{3 \alpha}}{\Gamma(3 \alpha+1)}+\cdots\right) \\
& =e^{(x+y) / 3} \sum_{i=0}^{\infty} \frac{(-1)^{j} t^{j \alpha}}{\Gamma(j \alpha+1)}=e^{(x+y) / 3} E_{\alpha}\left(-t^{\alpha}\right),
\end{aligned}
$$

where $E_{\alpha}\left(-t^{\alpha}\right)$ is the Mittag-Leffler function defined by Eq. (8). Taking $\alpha=1$ in Eq. (62) will yield the following result:

$$
\begin{aligned}
u(x, y, t) & =e^{(x+y) / 3}\left(1-t+\frac{t^{2}}{2 !}-\frac{t^{3}}{3 !}+\cdots\right) \\
& =e^{(1 / 3)(x+y)-t},
\end{aligned}
$$

which is the exact solution of classical biological population equation.

\section{Numerical results and discussion}

To show the accuracy of the MGTFSM algorithm in handling examples provided, the two-dimensional plots of the 4th MGTFSM approximate solutions and exact solutions when $t \in[0,1]$ at different levels of fractional order $\alpha$ such that $\alpha=\{0.7,0.8,0.9,1\}$ are given in Figures 1-4, respectively. Tables 1-4 show the numerical values of the 4th MGTFSM approximate solutions the exact solutions and the absolute values of the errors for Examples 2-5, respectively, when $\alpha=\{0.7,0.8,0.9,1\}$ with some selected grid points $t$ with the step size 0.1 . From these figures and tables, it is clearly observed that when $\alpha \rightarrow 1$, the approximate solution obtained by the MGTFSM is very close to the exact solution, which indicates the accuracy and efficiency of the proposed method.

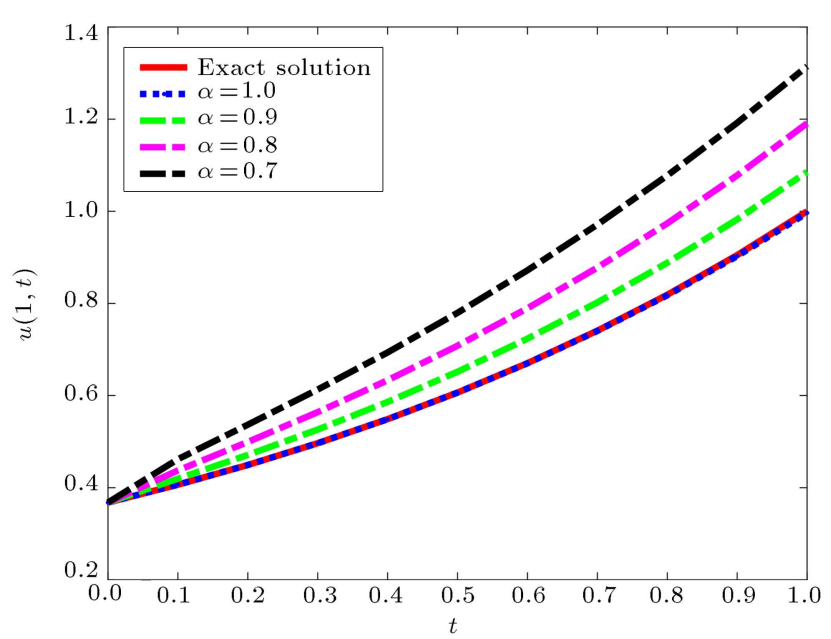

Figure 1. The behavior of the 4th Modified Generalized Taylor Fractional Series Method (MGTFSM) approximate solution and exact solution $u(1, t)$ for Example 2 .

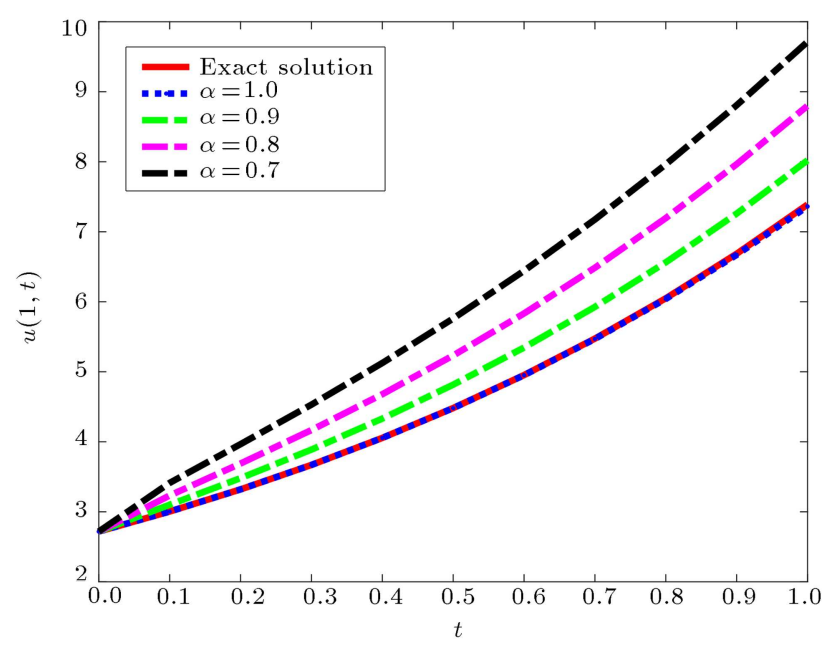

Figure 2. The behavior of the 4th Modified Generalized Taylor Fractional Series Method (MGTFSM) approximate solution and exact solution $u(1, t)$ for Example 3 .

\section{Conclusion}

In this paper, a new approximate analytical method called the Modified Generalized Taylor Fractional Series Method (MGTFSM) was successfully applied to obtain the numerical solutions of general nonlinear time-fractional partial differential equations with arbitrary nonlinear terms. The method was applied to many numerical examples. The results showed that the MGTFSM was an efficient and convenient method for finding numerical solutions to these problems. The obtained approximate solutions using the proposed method were in excellent agreement with the corresponding exact solutions. Finally, it can be concluded that the MGTFSM was powerful and efficient in finding approximate and analytical solutions to wider classes of nonlinear fractional problems.

In future works, we will extend this approach 


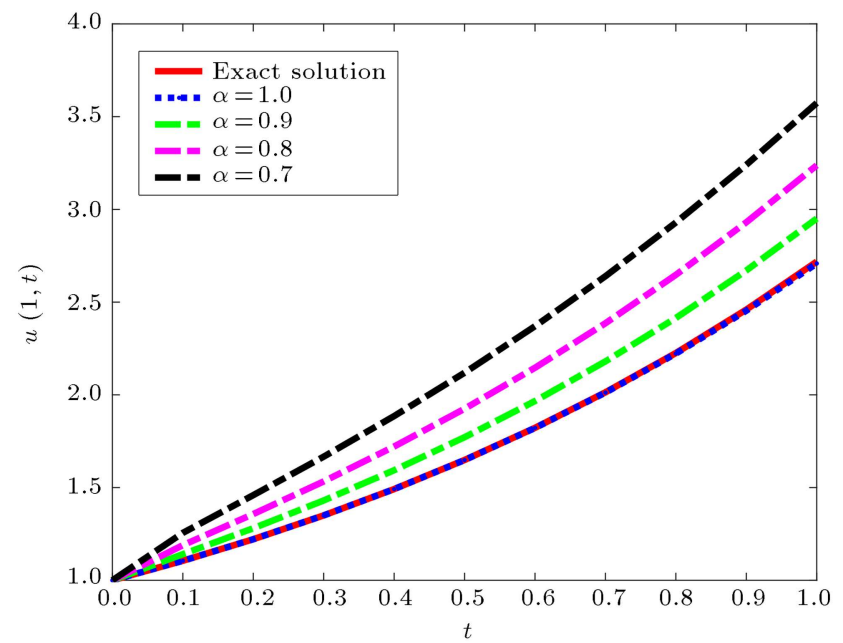

Figure 3. The behavior of the 4th Modified Generalized Taylor Fractional Series Method (MGTFSM) approximate solution and exact solution $u(1, t)$ for Example 4 .

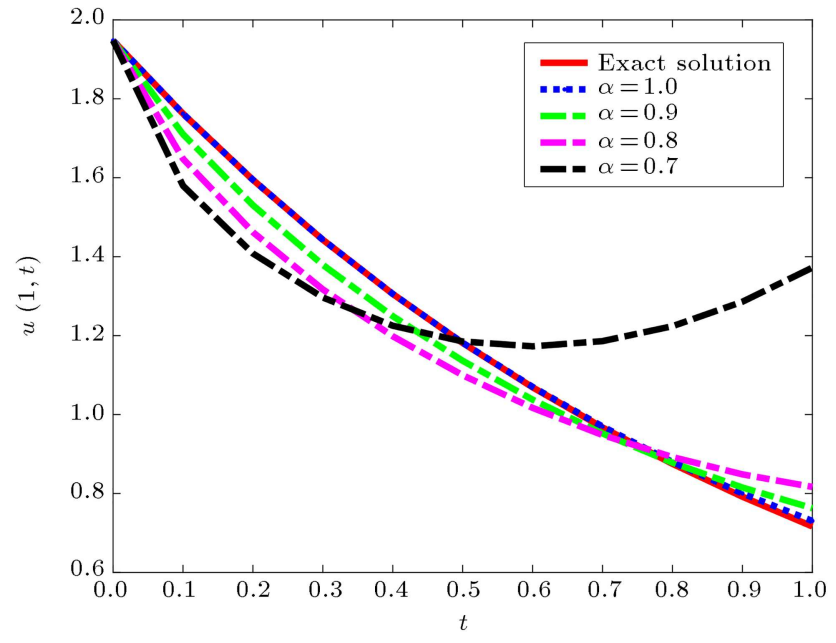

Figure 4. The behavior of the 4th Modified Generalized Taylor Fractional Series Method (MGTFSM) approximate solution and exact solution $u(1,1, t)$ for Example 5 .

Table 1. The numerical values of the 4th Modified Generalized Taylor Fractional Series Method (MGTFSM) approximate solution, exact solution $u(1, t)$, and the absolute value of the error for Example 2.

\begin{tabular}{lcccccc}
\hline $\boldsymbol{t}$ & $\boldsymbol{\alpha}=\mathbf{0 . 7}$ & $\boldsymbol{\alpha}=\mathbf{0 . 8}$ & $\boldsymbol{\alpha}=\mathbf{0 . 9}$ & $\boldsymbol{\alpha}=\mathbf{1}$ & Exact solution & $\left|\boldsymbol{u}_{\text {exact }}-\boldsymbol{u}_{M G T F S M}\right|$ \\
\hline 0.1 & 0.46191 & 0.43746 & 0.41969 & 0.40657 & 0.40657 & $3.1175 \times 10^{-8}$ \\
\hline 0.3 & 0.61312 & 0.56399 & 0.52622 & 0.49658 & 0.49659 & $7.8386 \times 10^{-6}$ \\
\hline 0.5 & 0.77963 & 0.70816 & 0.65172 & 0.60643 & 0.60653 & $1.0439 \times 10^{-4}$ \\
\hline 0.7 & 0.97106 & 0.87781 & 0.80211 & 0.74024 & 0.74082 & $1.9680 \times 10^{-6}$ \\
\hline 0.9 & 1.19200 & 1.07800 & 0.98249 & 0.90272 & 0.90484 & $2.1210 \times 10^{-3}$ \\
\hline
\end{tabular}

Table 2. The numerical values of the 4th Modified Generalized Taylor Fractional Series Method (MGTFSM) approximate solution, exact solution $u(1, t)$, and the absolute value of the error for Example 3 .

\begin{tabular}{lcccccc}
\hline $\boldsymbol{t}$ & $\boldsymbol{\alpha}=\mathbf{0 . 7}$ & $\boldsymbol{\alpha}=\mathbf{0 . 8}$ & $\boldsymbol{\alpha}=\mathbf{0 . 9}$ & $\boldsymbol{\alpha}=\mathbf{1}$ & Exact solution & $\left|\boldsymbol{u}_{\text {exact }}-\boldsymbol{u}_{M G T F S M}\right|$ \\
\hline 0.1 & 3.4130 & 3.2325 & 3.1011 & 3.0042 & 3.0042 & $2.3035 \times 10^{-7}$ \\
\hline 0.3 & 4.5304 & 4.1674 & 3.8882 & 3.6692 & 3.6693 & $5.7920 \times 10^{-5}$ \\
\hline 0.5 & 5.7607 & 5.2327 & 4.8156 & 4.4809 & 4.4817 & $7.7137 \times 10^{-4}$ \\
\hline 0.7 & 7.1752 & 6.4862 & 5.9269 & 5.4696 & 5.4739 & $4.3000 \times 10^{-3}$ \\
\hline 0.9 & 8.8079 & 7.9656 & 7.2597 & 6.6702 & 6.6859 & $1.5673 \times 10^{-2}$ \\
\hline
\end{tabular}

Table 3. The numerical values of the 4th Modified Generalized Taylor Fractional Series Method (MGTFSM) approximate solution, exact solution $u(1, t)$, and the absolute value of the error for Example 4.

\begin{tabular}{lcccccc}
\hline $\boldsymbol{t}$ & $\boldsymbol{\alpha}=\mathbf{0 . 7}$ & $\boldsymbol{\alpha}=\mathbf{0 . 8}$ & $\boldsymbol{\alpha}=\mathbf{0 . 9}$ & $\boldsymbol{\alpha}=\mathbf{1}$ & Exact solution & $\left|\boldsymbol{u}_{\text {exact }}-\boldsymbol{u}_{M G T F S M}\right|$ \\
\hline 0.1 & 1.2556 & 1.1892 & 1.1408 & 1.1052 & 1.1052 & $8.4742 \times 10^{-8}$ \\
\hline 0.3 & 1.6666 & 1.5331 & 1.4304 & 1.3498 & 1.3499 & $2.1308 \times 10^{-5}$ \\
\hline 0.5 & 2.1193 & 1.9250 & 1.7715 & 1.6484 & 1.6487 & $2.8377 \times 10^{-4}$ \\
\hline 0.7 & 2.6396 & 2.3861 & 2.1804 & 2.0122 & 2.0138 & $1.5819 \times 10^{-3}$ \\
\hline 0.9 & 3.2403 & 2.9304 & 2.6707 & 2.4538 & 2.4596 & $5.7656 \times 10^{-3}$ \\
\hline
\end{tabular}

Table 4. The numerical values of the 4th Modified Generalized Taylor Fractional Series Method (MGTFSM) approximate solution, exact solution $u(1,1, t)$, and the absolute value of the error for Example 5 .

\begin{tabular}{lcccccc}
\hline $\boldsymbol{t}$ & $\boldsymbol{\alpha}=\mathbf{0 . 7}$ & $\boldsymbol{\alpha}=\mathbf{0 . 8}$ & $\boldsymbol{\alpha}=\mathbf{0 . 9}$ & $\boldsymbol{\alpha}=\mathbf{1}$ & Exact solution & $\left|\boldsymbol{u}_{\text {exact }}-\boldsymbol{u}_{\text {MGT F } S M}\right|$ \\
\hline 0.1 & 1.2556 & 1.1892 & 1.1408 & 1.1052 & 1.1052 & $8.4742 \times 10^{-8}$ \\
\hline 0.3 & 1.6666 & 1.5331 & 1.4304 & 1.3498 & 1.3499 & $2.1308 \times 10^{-5}$ \\
\hline 0.5 & 2.1193 & 1.9250 & 1.7715 & 1.6484 & 1.6487 & $2.8377 \times 10^{-4}$ \\
\hline 0.7 & 2.6396 & 2.3861 & 2.1804 & 2.0122 & 2.0138 & $1.5819 \times 10^{-3}$ \\
\hline 0.9 & 3.2403 & 2.9304 & 2.6707 & 2.4538 & 2.4596 & $5.7656 \times 10^{-3}$ \\
\hline
\end{tabular}


to include another set of Nonlinear Fractional Partial Differential Equations (NFPDEs) with high-order fractional derivatives $n \alpha$, where $n-1<n \alpha \leq n$ and $n \in N^{*}$.

\section{Nomenclature}

$\begin{array}{ll}\text { MGTFSM } & \begin{array}{l}\text { Modified Generalized Taylor Fractional } \\ \text { Series Method }\end{array} \\ u(1, t) & \text { Exact solution } u(x, t) \text { at } x=1 \\ u(1,1, t) & \text { Exact solution } u(x, y, t) \text { at } x=y=1 \\ u_{\text {exact }} & \text { Exact solution } \\ u_{M G T F S M} & \text { 4th MGTFSM approximate solution } \\ \mid u_{\text {exact }}-u_{M G T F S M} & \text { Absolute error between } u_{\text {exact }} \text { and } \\ & u_{M G T F S M}\end{array}$

\section{Acknowledgments}

The authors thank the anonymous referees for their careful reading of the paper and valuable remarks that improved the final version of the paper.

\section{References}

1. Al-Ghafri, K.S. and Rezazadeh, H. "Solitons and other solutions of $(3+1)$-dimensional space-time fractional modified KdV-Zakharov-Kuznetsov equation", Applied Mathematics and Nonlinear Sciences, 4(2), pp. 289-304 (2019).

2. Asaduzzaman, M. and Ali, Z. "Existence of positive solution to the boundary value problems for coupled system of nonlinear fractional differential equations ", AIMS Mathematics, 4(3), pp. 880-895 (2019).

3. Benavides-Cruz, M., Calderón-Ramón, C., GómezAguilar J.F., et al. "Numerical simulation of metallic nanostructures interacting with electromagnetic fields using the Lorentz-Drude model and FDTD method", International Journal of Modern Physics C, 27(04), 1650043 (2016).

4. Ding, Q. and Wong, P.J.Y. "Quintic non-polynomial spline for time-fractional nonlinear Schrdinger equation", Advances in Difference Equations, 2020(46), pp. 1-27 (2020).

5. Dubey, V.P., Kumar, R., Kumar, D., Khan, I., and Singh, J. "An efficient computational scheme for nonlinear time fractional systems of partial differential equations arising in physical sciences", Advances in Difference Equations, 2020(577), pp. 1-27 (2020).

6. Farman, M., Akgül, A., Ahmad, A., and Imtiaz, S. "Analysis and dynamical behavior of fractional-order cancer model with vaccine strategy", Mathematical Methods in the Applied Sciences, 43, pp. 4871-4882 (2020).

7. Ghanbari, B. and Gómez-Aguilar, J.F. "Optical solution solutions for the nonlinear Radhakrishnan-KunduLakshmanan equation", Modern Physics Letters B, 33(32), 1950437 (2019).
8. Hosseini, K., Aligoli, M., Mirzazadeh, M., Eslami, M., and Gómez-Aguilar, J.F. "Dynamics of rational solutions in a new generalized Kadomtsev-Petviashvili equation ", Modern Physics Letters B, 33(35), 195043 (2020).

9. Kumar, D., Singh, J., Tanwar, K., and Baleanu, D. "A new fractional exothermic reactions model having constant heat source in porous media with power, exponential and Mittag-Leffler laws", International Journal of Heat and Mass Transfer, 138, pp. 12221227 (2019).

10. Kumar, S., Ghosh, S., Kumar, R., and Jleli, M. "A fractional model for population dynamics of two interacting species by using spectral and Hermite wavelets methods", Numerical Methods for Partial Differential Equations, 138, pp. 1-21 (2020).

11. Mohammadi, F., Moradi, L., Baleanu, D., and Jajarmi, A. "A hybrid functions numerical scheme for fractional optimal control problems: application to non-analytic dynamical systems", Journal of Vibration and Control, 24(21), pp. 5030-5043 (2018).

12. Osman, M.S., Ali, K.K., and Gómez-Aguilar, J.F. "A variety of new optical soliton solutions related to the nonlinear Schrdinger equation with time-dependent coefficients", Optik, 222, 165389 (2020).

13. Prakash, A., Prakash, D.G., and Veeresha, P. "A reliable algorithm for time-fractional Navier-Stokes equations via Laplace transform", Nonlinear Engineering, 8, pp. 695-701 (2019).

14. Sedeeg, A.K.H., Nuruddeen, R.I., and Gómez-Aguilar, J.F. "Generalized optical soliton solutions to the $(3+$ 1)-dimensional resonant nonlinear Schrdinger equation with Kerr and parabolic law nonlinearities ", Optical and Quantum Electronics, 51(6), P. 173 (2019).

15. Taneco-Hernndeza, M.A., Morales-Delgadob, V.F., and Gómez-Aguilar, J.F. "Fractional KuramotoSivashinsky equation with power law and stretched Mittag-Leffler kernel", Physica A: Statistical Mechanics and Its Applications, 527, 121085 (2019).

16. Thabet, H. and Kendre, S. "New modification of Adomian decomposition method for solving a system of nonlinear fractional partial differential equations", International Journal of Advances in Applied Mathematics and Mechanics, 6, pp. 1-13 (2019).

17. Sontakke, B., and Shelke, A.S. "Solution of time fractional partial differential equations by variational iteration method and applications", Asian Journal of Mathematics and Computer Research, 23(1), pp. 7-16 (2018).

18. Ali, A.H., Ahmed, A.B., and Mohamed, H. "Homotopy analysis method for solving some partial time fractional differential equation", IOSR Journal of Mathematics, 16(4), pp. 35-40 (2020).

19. Jleli, M., Kumar, S., Kumar, R., and Sameta, B. "Analytical approach for time fractional wave equations in the sense of Yang-Abdel-Aty-Cattani via the homotopy perturbation transform method", Alexandria Engineering Journal, 59, pp. 2859-2863 (2020). 
20. Khalouta, A. and Kadem, A. "Comparison of new iterative method and natural homotopy perturbation method for solving nonlinear time-fractional wavelike equations with variable coefficients", Nonlinear Dynamics and Systems Theory, 19(1-SI), pp. 160-169 (2019).

21. Khalouta, A. and Kadem, A. "Solutions of nonlinear time-fractional wave-like equations with variable coefficients in the form of Mittag-Leffler functions", Thai Journal of Mathematics, 18(1), pp. 411-424 (2020).

22. Odibat, Z. "Fractional power series solutions of fractional differential equations by using generalized Taylor series", Applied and Computational Mathematics, 19(1), pp. 47-58 (2020).

23. Khalouta, A. and Kadem, A. "A new modification of the reduced differential transform method for nonlinear fractional partial differential equations", Journal of Applied Mathematics and Computational Mechanics, 19(3), pp. 45-58 (2020).

24. Avci, I. and Mahmudov, N.I. "Numerical solutions for multi-term fractional order differential equations with fractional Taylor operational matrix of fractional integration", Mathematics, 8(1), pp. 1-24 (2020).

25. Khalouta, A. and Kadem, A. "Solution of the fractional Bratu-type equation via fractional residual power series method ", Tatra Mountains Mathematical Publications, 76, pp. 127-142 (2020).

26. Khalouta, A. and Kadem, A. "New analytical method for solving nonlinear time-fractional reactiondiffusion-convection problems", Revista Colombiana de Matemáticas, 54(1), pp. 1-11 (2020).

27. Kilbas, A.A., Srivastava, H.M., and Trujillo, J.J., Theory and Application of Fractional Differential equations, Elsevier, North-Holland (2006).

28. Podlubny, I., Fractional Differential Equations, Academic Press, New York (1999).

29. Riabi, L., Belghaba, K., Hamdi Cherif, M., and Ziane, Dj. "Homotopy perturbation method combined with
ZZ transform to solve some nonlinear fractional differential equations", International Journal of Analysis and Applications, 17(3), pp. 406-419 (2019).

30. Rao, T.R.R. "Numerical solution for time fractional gas dynamics equations through reduced differential transform coupled with fractional complex transform", International Journal of Pure and Applied Mathematics, 117(20), pp. 111-118 (2017).

31. Momani, S. and Yildirim, A. "Analytical approximate solutions of the fractional convection diffusion equation with nonlinnear source term by He's homotopy perturbation method", International Journal of Computer Mathematics, 87(5), pp. 1057-1065 (2010).

32. Singh, J., Kumar, D., and Kiliçman, A. "Numerical solutions of nonlinear fractional partial differential equations arising in spatial diffusion of biological populations", Abstract and Applied Analysis, Article ID 535793, pp. 1-12 (2014).

\section{Biographies}

Ali Khalouta received his $\mathrm{PhD}$ at Setif University (Algeria) in 2019. He is working at University of Sétif (Algeria) as an Associate Professor of Mathematics. His research areas include partial differential Equations, fractional calculus, numerical analysis of integrodifferential equations, and stochastic analysis.

Abdelouahab Kadem received his $\mathrm{PhD}$ at Metz University (France) in 1988 and his PhD at Setif University (Algeria) and Chalmers University of Thechnology Goteborg University (Sweden) in 2006. He is working at University of Setif (Algeria) as a Professor of Mathematics. His research areas include partial differential equations, fractional calculus, control theory, and numerical analysis of hyperbolic pdes and integrodifferential equations. 